\title{
Correlation of Albumin-Bilirubin (ALBI) Score with Child-Turcotte-Pugh (CTP) Score in the Evaluation of Liver Cirrhosis
}

\author{
Sabrina Shafiq ${ }^{1, *}$, Mohammad Nuruzzaman Khan², Bipasha Majumder', \\ Mohammad Shahinul Alam ${ }^{3}$, Mohammad Saiful Islam ${ }^{1}$, Tuhin Sultana ${ }^{1}$ \\ ${ }^{1}$ Department of Laboratory Medicine, Bangabandhu Sheikh Mujib Medical University (BSMMU), Dhaka, Bangladesh \\ ${ }^{2}$ Department of Neurosurgery, Shaheed Suhrawardy Medical College, Dhaka, Bangladesh \\ ${ }^{3}$ Department of Hepatology, Bangabandhu Sheikh Mujib Medical University (BSMMU), Dhaka, Bangladesh
}

Email address:

sabrinashafiq1980@gmail.com (S. Shafiq),drkhosru71@yahoo.com (M. N. Khan), bipashac21988@gmail.com (B. Majumder), shahinul67@yahoo.com (M. S. Alam),drsislambulbul@gmail.com (M. S. Islam),drtsultana@gmail.com (T. Sultana)

${ }^{*}$ Corresponding author

\section{To cite this article:}

Sabrina Shafiq, Mohammad Nuruzzaman Khan, Bipasha Majumder, Mohammad Shahinul Alam, Mohammad Saiful Islam, Tuhin Sultana. Correlation of Albumin-Bilirubin (ALBI) Score with Child-Turcotte-Pugh (CTP) Score in the Evaluation of Liver Cirrhosis. American Journal of Laboratory Medicine. Vol. 4, No. 3, 2019, pp. 60-64. doi: 10.11648/j.ajlm.20190403.12

Received: May 25, 2019; Accepted: June 20, 2019; Published: July 2, 2019

\begin{abstract}
Cirrhosis of liver is characterized by the replacement of normal liver tissue by scar tissue. The Child-TurcottePugh (CTP) score is used to assess the prognosis of cirrhosis and included five factors: total bilirubin level, serum albumin, prothrombin time, ascites and hepatic encephalopathy. CTP score depends on clinical assessment which may result variation in scoring. Recently a new score is introduced, Albumin-Bilirubin (ALBI) score which may be done instead of CTP scoring in cirrhotic patient. This cross sectional study was conducted at the Department of Laboratory Medicine in collaboration with the Department of Hepatology, BSMMU, Dhaka, from March 2018 to February 2019. Blood samples were assayed from eighty one diagnosed cirrhotic patients. For all statistical analysis SPSS version 22 was used. The mean age of the study group was $46.1 \pm 16.0$ years. $64.2 \%$ were male and $35.8 \%$ were female. Kappa $(\mathrm{k})$ value was 0.759 between ALBI and CTP score. Pearson's correlation coefficient (r) test showed significant strong positive correlation between CTP score and ALBI score $(\mathrm{r}=+0.853, \mathrm{p}<0.001)$. This study concluded that the ALBI score may be done alternative to the CTP score in cirrhotic patient because it's simple, two parameters only, more evidence based and more objective.
\end{abstract}

Keywords: Cirrhosis of Liver, Child-Turcotte-Pugh Score, Albumin-Bilirubin Score

\section{Introduction}

Cirrhosis is a diffuse process of fibrosis with nodule formation [1]. Cirrhosis is caused by viruses, alcohol, nonalcoholic steatohepatitis (NASH) and metabolic causes including autoimmune conditions [2]. 20-30\% of chronic hepatitis $\mathrm{B}$ and $15-30 \%$ of chronic hepatitis $\mathrm{C}$ patients develop cirrhosis [3]. InBangladesh 8.5 million adults are infected with chronic hepatitis B virus (HBV) and $61.15 \%$ of cirrhosis is caused by HBV $[4,5]$. HCV is the second cause of chronic liver disease in Bangladesh [5]. NAFLD is also contributor of cirrhosis in Bangladesh [6].
Diagnosis of cirrhosis is based on blood tests, medical imaging, endoscopic examination and histopathology. Biopsy is avoided as most of the cirrhotic patient has prolonged prothrombin time that may cause intraperitoneal bleeding [7]. Toprevent biopsy related complications now a day's cirrhosis is diagnosed solely by clinical evidence, medical imaging, fibroscan and endoscopy of upper gastrointestinal tract.

Multiple scoring systems are available for assessing liver function and the severity of liver injury, including the Child-Turcotte-Pugh (CTP) score, the Model for End-Stage 
Liver Disease (MELD) score andthe MELD-Sodium (MELD-Na) score [2]. The Child-Turcotte-Pugh (CTP) score is widely used for the severity of hepatic dysfunction in patients with cirrhosis. Beside these scores, recently a new scoring is introduced, namely the Albumin-Bilirubin (ALBI) score [8]. The CTP score includes five parameters, two clinical and three laboratory parameters including ascites, hepatic encephalopathy, prothrombin time or INR, serum albumin and serum bilirubin level. The CTP scoring system, with a total of 15 points, classifies patients into class A (5-6 points), B (7-9 points) and C (10-15 points) [9].

Compared to CTP score, there are several advantages of the Albumin-Bilirubin (ALBI) score in grading of liver cirrhosis. First, the two parameters, serum albumin and serum bilirubin for the ALBI score, are obtained by accessible blood tests. But the clinical assessment of ascites and hepatic encephalopathy for the CTP score is difficult to score consistently. Moreover, different grades of ascites are not uniform in CTP score $[9,10]$. Second, the ALBI score is selected on the basis of a mathematical model but the five components of the CTP score are selected empirically [11]. Third, the values of bilirubin in the CTP score should be modified for chronic cholestatic diseases such as primary biliary cirrhosis for better result [12]. Fourth, ALBI score predicted survival of HBV related cirrhotic patient at 12, 24 and 36 months with an accuracy of $78.7 \%, 83.0 \%$ and $83.3 \%$, respectively which is better than CTP score [13].

For cirrhotic patient the sensitivity and specificity of CTP score is $90 \%$ and $33 \%$ but for ALBI score is $84 \%$ and $39 \%$ [14]. Another study support that sensitivity and specificity of ALBI score in liver cirrhosis patient with upper gastrointestinal bleeding is $92.86 \%$ and $64.01 \%$ respectively but in case of CTP score sensitivity and specificity is $57.14 \%$ and $86.24 \%$ respectively. However in HBV related liver cirrhosis sensitivity and specificity of ALBI score is $100.00 \%$ and $66.67 \%$ respectively but CTP score is $88.89 \%$ and $64.94 \%$ respectively. These values have been evaluated in hospital death of cirrhotic patient in china [15]. Considering the above scenario, the newly developed ALBI score is more convenient than CTP score.

Albumin-Bilirubin score are simple, repeatable, less expensive, can be done easily even in peripheral laboratory, without the need for special tests. This new score may be a better tool than CTP score in cirrhotic patients for early management and will be helpful for quick decision making for clinician in near future and beneficial for cirrhotic patients.

\section{Materials and Methods}

This cross sectional study was carried out in the department of Laboratory Medicine and Hepatology, BSMMU from March 2018 to February 2019. Purposive sampling with set inclusion criteria was followed. Total 81 diagnosed cases of cirrhotic patients (27 in each class of CTP score) were included in this study. Patients were diagnosed according to history, clinical sign and symptom.
By standard venipuncture procedure a total of $5.0 \mathrm{ml}$ of venous blood was collected from median cubital vein after asepsis with $0.5 \%$ chlorhexidine gluconate. Among $5 \mathrm{ml}$, about $2.0 \mathrm{ml}$ blood was taken into plastic red screw-capped plain tube for albumin and bilirubin tests and $3.0 \mathrm{ml}$ blood was taken in a blue screw capped citrate tube at 1: 9 dilutions for prothrombin time. The collected tubes were labeled with the patient's identification number. Universal precaution was maintained. Serum albumin and bilirubin were estimated by using photometric principle method and these tests were done by biochemistry auto- analyzer (SIEMENS Dimension RxL Max, USA) in the Laboratory Medicine Department, BSMMU, Dhaka. Prothrombin time was done in automated coagulometer (Sysmex CA-500 series). Normal value: Serum albumin $-3.5-5 \mathrm{~g} / \mathrm{dl}$, Serum bilirubin- 3-16 $\mu \mathrm{mol} / \mathrm{L}$, Prothrombin time- 10.5-13.5 seconds [16]. Then CTP and ALBI scores were calculated for each patient and correlated.

\section{Data analysis}

All data were analyzed using statistical package for social science (SPSS) version 22.

CTP score calculation:

Table 1. Interpretation of CTP score-.

\begin{tabular}{llll}
\hline Measure & 1 point & 2 points & 3 points \\
\hline Total bilirubin $(\mu \mathrm{mol} / \mathrm{l})$ or & $<34$ & $34-50$ & $>50$ \\
$(\mathrm{mg} / \mathrm{dl})$ & $(<2)$ & $(2-3)$ & $(>3)$ \\
Serum albumin $(\mathrm{g} / \mathrm{dl})$ or & $>3.5$ & $2.8-3.5$ & $<2.8$ \\
$(\mathrm{~g} / \mathrm{l})$ & 35 & $28-35$ & $<28$ \\
Prothrombintime, & $<4.0$ & $4.0-6.0$ & $>6.0$ \\
prolongation $(\mathrm{s})$ or INR & $<1.7$ & $1.7-2.3$ & $>2.3$ \\
& & Mild (or suppressed & $\begin{array}{l}\text { Moderate to } \\
\text { severe (or } \\
\text { Ascites }\end{array}$ \\
& None & with medication) & $\begin{array}{l}\text { refractory) } \\
\text { Grade 3-4 }\end{array}$ \\
\hline Hepatic encephalopathy & None & Grade 1 -2 &
\end{tabular}

CTP class A (5-6 points), class B (7-9 points) and class C (10-15 points) [17].

\section{ALBI score calculation:}

The ALBI score was calculated from the following formula - ALBI score $=-0.085 \times($ albumin $[\mathrm{g} / \mathrm{L}])+0.66 \times$ $\log 10$ (total bilirubin $[\mu \mathrm{mol} / \mathrm{L}])$ and was classified as- grade 1 $(\leq-2.60)$, grade $2(>-2.60$ to $\leq-1.39)$, or grade $3(>-1.39)$ [8].

\section{Results}

This cross sectional study was carried out in the department of Laboratory Medicine and department of Hepatology, BSMMU, Dhaka. The main objective of the study was to assess the correlation of ALBIscore with CTP score in cirrhotic patient of Bangladesh. Table 4 shows correlation between the ALBI and CTP score. Kappa test were done to see the relationship between CTP score and ALBI grade in cirrhotic patients. The test of significance was calculated and $\mathrm{p}$ value $<0.001$ was accepted as level of significance. 
Table 2. Age distribution of the study patient $(n=81)$.

\begin{tabular}{lll}
\hline Age of the patient (in years) & No of the Patient & Percentage (\%) \\
\hline $21-30$ & 14 & 17.3 \\
$31-40$ & 21 & 25.9 \\
$41-50$ & 19 & 23.5 \\
$51-60$ & 13 & 16.0 \\
$61-70$ & 10 & 12.3 \\
$71-80$ & 2 & 2.5 \\
$81-90$ & 2 & 2.5 \\
Total & 81 & 100.0 \\
\hline
\end{tabular}

Mean \pm SD: 46.1 \pm 16.0 . Range (min-max): 21 - 90 years.

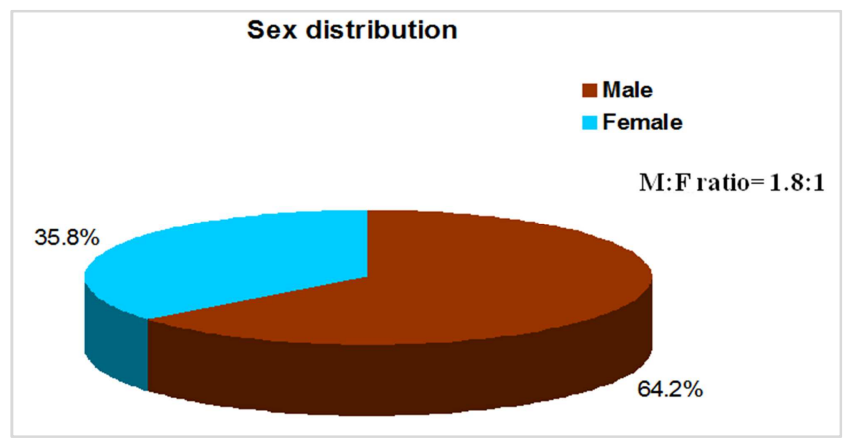

Figure 1. Pie diagram showing the sex distribution of the patients $(n=81)$.

Table 3. Laboratory investigations among the study patient $(n=81)$.

\begin{tabular}{lll}
\hline Variables & Range & Mean \pm SD \\
\hline Serum albumin $(\mathrm{g} / \mathrm{dl})$ & $1.30-4.80$ & $3.07 \pm 0.90$ \\
Serum bilirubin $(\mathrm{mg} / \mathrm{dl})$ & $0.20-29.60$ & $4.15 \pm 6.51$ \\
Prothrombin Time $(\mathrm{sec})$ & $10.00-47.80$ & $16.44 \pm 4.80$ \\
$\mathrm{INR}$ & $0.83-4.09$ & $1.39 \pm 0.41$ \\
\hline
\end{tabular}

Table 4. Association of CTP (Child-Turcotte-Pugh) score with ALBI grade $(n=81)$.

\begin{tabular}{lllllll}
\hline & n & \multicolumn{2}{l}{ ALBI Grade } & Kappa & p-value \\
\hline Class A & 27 & $23(85.2 \%)$ & $4(14.8 \%)$ & $0(0.0 \%)$ & & \\
Class B & 27 & $0(0.0 \%)$ & $21(77.8 \%)$ & $6(22.2 \%)$ & 0.759 & $<0.001^{\text {s }}$ \\
Class C & 27 & $0(0.0 \%)$ & $3(11.1 \%)$ & $24(88.9 \%)$ & & \\
Total & 81 & $23(28.4 \%)$ & $28(34.6 \%)$ & $30(37.0 \%)$ & & \\
\hline
\end{tabular}

$\mathrm{s}=$ significant

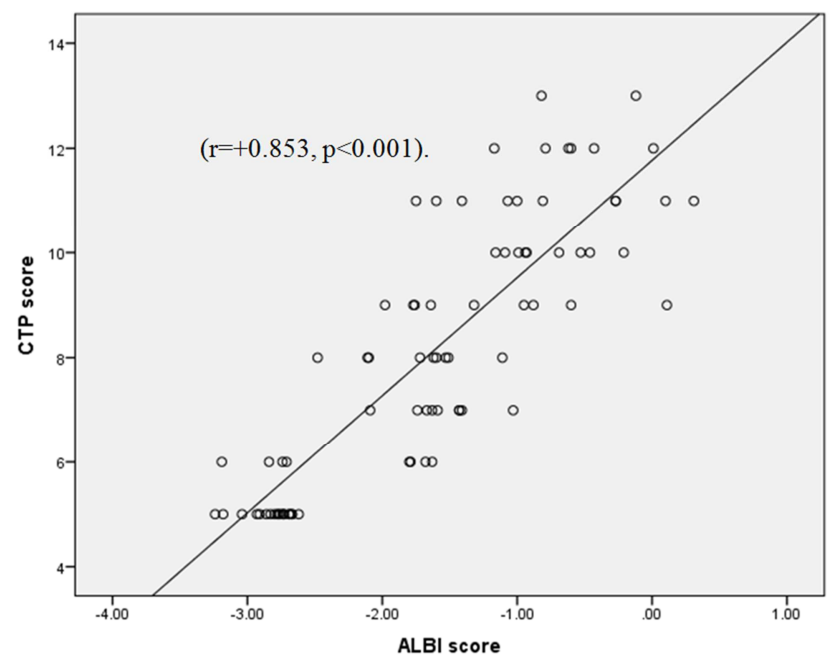

Figure 2. Scatter diagram showing correlation between CTP score and ALBI score $(n=81)$.

\section{Discussion}

Cirrhosis of liver is a major public health issue worldwide because of its increased mortality and morbidity rates. Multiple scoring systems are available for evaluation of cirrhotic patient. In this cross sectional study, the Child-Turcotte-Pugh score was correlated with newly developed Albumin-Bilirubin score. A total of 81 clinically diagnosed cirrhotic patients were selected from the department of Hepatology, BSMMU. The study was carried out in the department of Laboratory Medicine, BSMMU.

In our study, it was observed in table 2 that the age range was 21-90 years and the highest number of patients $(25.9 \%)$ was 31-40 years. The mean age was $46.1 \pm 16.0$. The average age of patients of chronic liver disease was 51.95 years in Bangladesh [6]. In another study the mean age of liver cirrhosis was $49 \pm 11.13$ years [18]. This finding is nearly consistent with our study.

In this study, analysis of gender distribution in figure 1 showed among 81 patients $52(64.2 \%)$ patients were male and $29(35.8 \%)$ patients were female. Male and female ratio was 1.8:1. Males suffer more from cirrhosis of liver. Among gender distribution in liver cirrhosis patient of Bangladesh, $68.18 \%$ were male and $31.82 \%$ were female [19]. These findings are consistent with our study.

In the current study, themean $\pm \mathrm{SD}$ of laboratory variables in table 3 like serum albumin $(\mathrm{g} / \mathrm{dl})$, serum bilirubin $(\mathrm{mg} / \mathrm{dl})$, prothrombin time $(\mathrm{sec})$ and INR was found $3.07 \pm 0.90$, $4.15 \pm 6.51,16.44 \pm 4.80$ and $1.39 \pm 0.41$ respectively. The mean \pm $\mathrm{SD}$ of serum albumin $(\mathrm{g} / \mathrm{l})$, serum bilirubin $(\mathrm{mg} / \mathrm{dl})$, prothrombin time $(\mathrm{sec})$ and INR were $28.2 \pm 7.6,30.2 \pm 27.1$, $67.4 \pm 14.8,1.4 \pm 0.3$ respectively in a study in china [13]. This result is not consistent with the present study. In a study observed among 111 cirrhotic patients during assessing prognostic performance of ALBI score in Portugal among other scores, the mean \pm SD of serum albumin $(\mathrm{g} / \mathrm{dl})$, serum bilirubin $(\mathrm{mg} / \mathrm{dl})$, prothrombin time $(\mathrm{sec})$ and INR were $2.6 \pm 0.6,2.5 \pm 2.4,17.5 \pm 3.9,1.6 \pm 0.4$ respectively [20]. These findings are nearly similar with our study.

In this study, we observed in table 4 that CTP class A patients, 23 (85.2\%) had ALBI grade 1 and 4 (14.8\%) ALBI grade 2 patients with no ALBI grade 3 patients. In CTP class B, $21(77.8 \%)$ patients had ALBI grade 2 and $6(22.2 \%)$ ALBI grade 3 patients. In CTP class C, 24 (88.9\%) patients had ALBI grade 3 and 3 (11.1\%) ALBI grade 2 patients. There was no ALBI grade 1 patient both in CTP class B and C. Some patients of ALBI grade not correspond with CTP class as it depends on ascites and hepatic encephalopathy. Clinical diagnosis of ascites about one third cirrhotic patient are incorrect [21].

Moreover, hepatic encephalopathy easily missed in cirrhotic patient because clinical signs are mostly occult [22]. But ALBI grade well correlated with CTP score in the present study. While increasing CTP score, ALBI grade also increasing simultaneously. In a study among 89 patients in Japan, majority of ALBI grade 1 (14 of 15 patients: 93\%) had 
a CTP score of 5 (CTP class A) and $83 \%$ of ALBI grade 2 matched with CTP score 5, 6, 7, 8 that is both CTP class A and B. No patient was graded as ALBI grade 3 [23].

During observation of 100 patients in a study for predicting the in-hospital mortality in cirrhotic patients with acute-on-chronic liver failure in china nearly all patients had CTP class C (97.0\%), only 3patients had CTP class B (3.0\%) and all patients had ALBI grade $3(89.0 \%)$ and 11 patients had ALBI grade $2(11.0 \%)$ [14]. Incorporating CTP class C with ALBI grade 3, our result corresponds with this study. Another study done on 152 patients and correlate between ALBI grade and CTP score where 60 (39.5\%) patients were ALBI grade 1, $86(56.6 \%)$ patients were ALBI grade 2 and remaining $6(3.9 \%)$ were ALBI grade 3. Most patients of ALBI grade 1 had CTP score A. The majority (27 of 33) of CTP class B patients were ALBI grade 2 and all ALBI grade 3 patients were also CTP class B [24]. While correlating ALBI grade 1 and 2 with CTP class $\mathrm{A}$ and $\mathrm{B}$, our result nearly similar with this study.

Pearson's correlation coefficient (r) test was done to compare relationship between CTP score and ALBI score in figure 2 . In the present study, $r$ value showed +0.853 that suggested, significant strong positive correlation between CTP score and ALBI score in cirrhotic patients $(p<0.001)$. The ALBI score was positively correlated with the CTP score $(r=0.790, p<0.001)$ [25]. Positive correlations was also found between the ALBI score and the CTP score $(\mathrm{r}=$ $+0.576, \mathrm{p}<0.001$ ) [26]. Strong positive correlation between the ALBI and the CTP score $(r=0.759, \mathrm{p}<0.01)$ was evident [20]. These results are consistent with the current study.

According to the present study, observed ALBI score well correlated with CTP score. The ALBI score is quickly obtained by doing easily accessible routine blood tests for cirrhotic patients and rapidly calculated at bedside. This new score offers numerous benefits over the CTP score. Our data suggests that, the ALBI score may substitute the CTP score in the evaluation of cirrhotic patient.

\section{Conclusion}

This study revealed that the ALBI score was positively correlated with the CTP score in the evaluation of cirrhotic patient. It was found that increased ALBI score was associated with increased CTP score. ALBI score is easily done by estimating simply serum albumin and serum bilirubin tests. Therefore, ALBI score may be used as a new score instead of CTP score for the early detection of cirrhotic patient in daily clinical practice and will be helpful for clinician for better management.

\section{Acknowledgements}

The authors thanks to Department of Public Health and Informatics, BSMMU, Dhaka, Bangladesh for valuable suggestions about the statistical analyses.

\section{Compliance with Ethical Standards}

\section{Funding}

This study was not funded.

\section{Conflict of Interest}

None.

\section{Ethical Approval}

All procedures performed in studies involving human participants were in accordance with the ethical standards of the Institutional Review Board (IRB) of BSMMU, Dhaka, Bangladesh.

\section{Informed Consent}

Informed consent was obtained from each of the included participants. Strict confidentiality was maintained regarding the information of the patients.

\section{References}

[1] Kamath PS, Shah, VH. Overview of Cirrhosis, Sleisenger and Fordtran's Gastrointestinal and Liver Disease, Pathophysiology, Diagnosis, Management. $10^{\text {th }}$ ed, 1976; 1254-1260.

[2] McCormick PA. Hepatic Cirrhosis, Sherlock's Diseases of the Liver and Biliary System. $12^{\text {th }}$ ed, 2011; 103-118.

[3] World Health Organization. HepatitisB. 2018. (https://www.who.int/news-room/fact-sheets/detail/hepatitis-b). n

[4] Alam S, Azam G, Mustafa G, Alam M, Ahmad N. Past, present, and future of hepatitis B and fatty liver in Bangladesh Gastroenterol Hepatol Open Access. 2017; 6 (3): 197.

[5] Afroz S, Mahtab MA, Rahman S, Khan M. Hepatitis B virus is the leading cause of cirrhosis of liver in Bangladesh. Hepatol Int. 2007; 1 (1): 120.

[6] Rahman S, Ahmed MF, Alam MJ. Distribution of liver disease in Bangladesh: a cross-country study. Euroasian journal of hepato-gastroenterology. 2014; 4 (1): 25-30.

[7] Kalambokis G, Manousou P, Vibhakorn S, Marelli L, Cholongitas E, Senzolo M. Transjugular liver biopsy-indications, adequacy, quality of specimens, and complications- a systematic review. Journal of Hepatology. 2007; 47 (2): 284-294.

[8] Johnson PJ, Berhane S, Kagebayashi C, Satomura S, Teng M, Reeves HL, O'Beirne J, Fox R, Skowronska A, Palmer D, Yeo W. Assessment of liver function in patients with hepatocellular carcinoma: a new evidence-based approach - the ALBI grade. Journal of Clinical Oncology. 2015; 33 (6): 550-558.

[9] Infante-Rivard C, Esnaola S, Villeneuve JP. Clinical and statistical validity of conventional prognostic factors in predicting short-term survival among cirrhotics. Hepatology. 1987; 7 (4): 660-4. 
[10] Pugh RN, Murray-Lyon IM, Dawson JL, Pietroni MC, Williams R. Transection of the oesophagus for bleeding oesophageal varices. Br J Surg. 1973; 60: 646-9.

[11] Arroyo V. Pathophysiology, diagnosis and treatment of ascites in cirrhosis. Annals of hepatology. 2002; 1 (2): 72-9.

[12] Chan AW, Chan RC, Wong GL, et al. Evaluation of histological staging systems for primary biliary cirrhosis: correlation with clinical and biochemical factors and significance of pathological parameters in prognostication. Histopathology. 2014; 65: 174-86.

[13] Chen RC, Cai YJ, Wu JM, Wang XD, Song M, Wang YQ, Zheng MH, Chen YP, Lin Z, Shi KQ. Usefulness of albuminbilirubin grade for evaluation of long-term prognosis for hepatitis B-related cirrhosis. Journal of viral hepatitis. 2017; 24 (3): 238-45.

[14] Peng Y, Qi X, Tang S, Deng H, Li J, Ning Z, Dai J, Hou F, Zhao J, Wang R, Guo X. Child-Pugh, MELD, and ALBI scores for predicting the in-hospital mortality in cirrhotic patients with acute-on-chronic liver failure. Expert review of gastroenterology \& hepatology. 2016; 10 (8): 971-80.

[15] Zou D, Qi X, Zhu C, Ning Z, Hou F, Zhao J, Peng Y, Li J, Deng H, Guo X. Albumin-bilirubin score for predicting the inhospital mortality of acute upper gastrointestinal bleeding in liver cirrhosis: A retrospective study. Turk J Gastroenterol. 2016 Mar 1; 27 (2): 180-86.

[16] Walker SW. Laboratory reference ranges. Davidson's Principles Practice of Medicine, $21^{\text {st }}$ ed, 2010; 1293-1298.

[17] Cholongitas E, Papatheodoridis GV, Vangeli M, Terreni N, Patch D, Burroughs AK. Systematic review: the model for end-stage liver disease-should it replace Child-Pugh's classification for assessing prognosis in cirrhosis?. Alimentary pharmacology \& therapeutics. 2005; 22 (11-12): 1079-89.

[18] Lei Q, Zhang Y, Ke C, Yan C, Huang P, Shen H, Lei H, Chen Y, Luo J, Meng Z. Value of the albumin-bilirubin score in the evaluation of hepatitis B virus-related acute-on-chronic liver failure, liver cirrhosis, and hepatocellular carcinoma. Experimental and therapeutic medicine. 2018; 15 (3): 3074-9.
[19] Das DC, Al Mahtab M, Rahim MA, Malakar D, Kabir A, Rahman S. Hepatitis B virus remains the leading cause of cirrhosis of liver in Bangladesh. Bangladesh Medical Journal. 2016; 45 (3): 164-6.

[20] Xavier SA, Vilas-Boas R, Boal Carvalho P, Magalhães JT, Marinho CM, Cotter JB. Assessment of prognostic performance of Albumin-Bilirubin, Child-Pugh, and Model for End-stage Liver Disease scores in patients with liver cirrhosis complicated with acute upper gastrointestinal bleeding. European journal of gastroenterology \& hepatology. 2018; 30 (6):652-8.

[21] Khalid SK, Garcia-Tsao G, Sanyal AJ, Shah V. Ascites. Clinical features, diagnosis and natural history. Portal Hypertension. 2005: 285-99.

[22] Morgan, M. Y. Hepatic Encephalopathy in patients with cirrhosis, Sherlock's Diseases of the Liver and Biliary System. $12^{\text {th }}$ ed, $2011 ; 121-151$.

[23] Ogasawara S, Chiba T, Ooka Y, Suzuki E, Kanogawa N, Saito T, Motoyama T, Tawada A, Kanai F, Yokosuka O. Liver function assessment according to the Albumin-Bilirubin (ALBI) grade in sorafenib-treated patients with advanced hepatocellular carcinoma. Investigational new drugs. 2015; 33 (6): 1257-62.

[24] Lo CH, Liu MY, Lee MS, Yang JF, Jen YM, Lin CS, Chao HL, Shen PC, Huang WY. Comparison between ChildTurcotte-Pugh and albumin-bilirubin scores in assessing the prognosis of hepatocellular carcinoma after stereotactic ablative radiation therapy. International Journal of Radiation Oncology* Biology* Physics. 2017; 99 (1): 145-52.

[25] Chan AW, Chan RC, Wong GL, Wong VW, Choi PC, Chan HL, To KF. New simple prognostic score for primary biliary cirrhosis: Albumin-bilirubin score. Journal of gastroenterology and hepatology. 2015; 30 (9): 1391-6.

[26] Chen B, Lin S. Albumin-bilirubin (ALBI) score at admission predicts possible outcomes in patients with acute-on-chronic liver failure. Medicine. 2017; 96 (24). 